\title{
Centralized Quasi-Static Channel Assignment for Multi-Radio Multi-Channel Wireless Mesh Networks
}

\author{
Juan REN, Zhengding QIU \\ Institute of Information Science, Beijing Jiaotong University, Beijing, China \\ Email: \{04112047, zdqiu\}@bjtu.edu.cn \\ Received February 19, 2009; revised March 19, 2009; accepted March 20, 2009
}

\begin{abstract}
Employing multiple channels in wireless multihop networks is regarded as an effective approach to increasing network capacity. This paper presents a centralized quasi-static channel assignment for multi-radio multi-channel Wireless Mesh Networks (WMNs). The proposed channel assignment can efficiently utilize multiple channels with only 2 radios equipped on each mesh router. In the scheme, the network end-to-end traffics are first modeled by probing data at wireless access points, and then the traffic load between each pair of neighboring routers is further estimated using an interference-aware estimation algorithm. Having knowledge of the expected link load, the scheme assigns channels to each radio with the objective of minimizing network interference, which as a result greatly improves network capacity. The performance evaluation shows that the proposed scheme is highly responsive to varying traffic conditions, and the network performance under the channel assignment significantly outperforms the single-radio IEEE 802.11 network as well as the 2-radio WMN with static 2 channels.
\end{abstract}

Keywords: Wireless Mesh Networks, Multihop Network, Channel Assignment, Multi-Radio

\section{Introduction}

WMN [1] is a promising wireless technology for numerous applications, e.g., broadband home networking, community and neighborhood networks, enterprise networking, building automation, etc. [2,3]. However, interference among wireless links significantly impacts the performance of WMNs. As a multi-hop wireless network, the actual goodput available to WMN applications decreases a lot when forwarding or relaying packets over multiple wireless hops.

Fortunately, the IEEE 802.11 PHY specification permits simultaneous operation of multiple non-overlapping channels. By deploying multi-radio routers in infrastructure-based networks and assigning radios to non-overlapping channels, the routers can communicate simultaneously with little interference in spite of being in direct interference range of each other. Therefore, the capacity

* This work is supported by National Basic Research Program of China (973 Program) (2007CB307100). of wireless networks can be increased. While due to the limited number of channels available, the interference cannot be completely eliminated. In addition, the channel assignment must be restricted to the number of radios on each wireless node. So it's a challenging problem deserving our research.

In equipping routers with multiple radios, a naive strategy would be to equip each router with the number of radios equal to the number of orthogonal channels. However, this strategy is economically prohibitive due to the significant number of non-overlapping channels. Another channel assignment strategy is to frequently change channel on the interface, for instance, for each packet transmission based on current state of the medium. Such dynamic channel assignment approaches [4-6] require channel switching at a very fast time scale (per packet or a handful of packets). The fast-channel switching requirement makes these approaches unsuitable for use with commodity hardware, where channel switching delays itself can be in the order of milliseconds [4]. Some of the dynamic channel assignment approaches also re- 
quire specialized MAC protocols or extensions of 802.11 MAC layer, making them further unsuitable for use in commodity 802.11 hardware.

In order to use multiple channels with commodity hardware, several researches [7-9] focused on developing techniques that assign channels statically. Such static assignments can be changed whenever there are significant changes to traffic load or network topology. Since WMN is an infrastructured network and aims to provide reliable broadband services, such changes are infrequent enough that the channel-switching delay and traffic measurement overheads are insignificant. We refer to the above as quasi-static channel assignments. However, most of the existing quasi-static channel assignments are performed offline and bound with routing.

In this paper, we address the problem of quasi-static channel assignment independent of routing. A centralized quasi-static channel assignment algorithm is proposed in the context of networks with multi-radio nodes. In the channel assignment, we use a novel scheme to estimate the traffic load on each wireless link. The estimation considers the traffic on the link itself as well as the interfering traffics introduced by its neighbors. Having knowledge of the expected load on each link, the algorithm can intelligently select different channels for each radio with the objective of minimizing network interference, which as a result efficiently improves the network capacity. To evaluate the algorithm performance, a corresponding channel assignment protocol is implemented in ns-2 simulations [10] and we incorporate the well-known WCETT (Weighted Cumulative Expected Transmission Time) path metric [11], which is tailored for multi-radio multihop wireless networks, into the AODV (Ad Hoc On Demand Distance Vector) routing protocol [12] as our multi-radio routing protocol. The performance evaluation shows that the proposed scheme is highly responsive to varying traffic conditions, and the network performance under the channel assignment significantly outperforms the single-radio IEEE 802.11 network as well as the 2-radio WMN with static 2 channels.

The rest of the paper is organized as follows. Section 2 gives the system architecture of the proposed multi-radio WMN. In Section 3, we describe the centralized quasi-static channel assignment scheme. In Section 4, we evaluate the performance of our channel assignment algorithm using the ns- 2 simulations. Section 5 concludes the paper.

\section{System Architecture}

In this section, we formulate the interference problem involved in wireless multihop networks and present the architecture of multi-radio multi-channel WMNs to resolve this problem.

\subsection{Interference Problem}

Traditional 802.11-based wireless networks can't transmit data simultaneously as wired networks because of the intra-path and inter-path interference. For example in Figure 1, although the two flows transmit separately on path 1 and path 2, nodes must compete with each other for a common channel, which reduces network throughput hardly. If node 3 is in transmission, all the nodes in interference range of node 3 should keep silence, or a collision will occur. In contrast, if we assign interfering hops to different channels, then one collision domain can be broken into several collision domains with each operating in a different frequency range. When the ingress-egress node pairs that originally pass through the collision domain now take different paths to route their traffic, hops using different channels can transmit simultaneously and the network throughput will increase.

\subsection{Multi-Radio Multi-Channel WMN Architecture}

Figure 2 gives the architecture of multi-radio multi-channel WMN. The wireless mesh backbone network consists of mesh routers (MR), mesh access routers (MAR) and the gateway. Mesh routers provide purely wireless routing services. Mesh access routers provide not only wireless routing services but also wireless access services. Each WMN has at least one gateway, which can also be served as the access point for wireless users. The integration of WMN with other networks such as the Internet, cellular, IEEE 802.11, IEEE 802.15, IEEE 802.16, sensor networks, etc., can be accomplished through the gateway and bridging functions in the mesh routers.

In WMN only end users may frequently move and mesh backbone facilities are almost static once been settled. In addition, both the gateway and the mesh access routers have aggregation capability. So we use them to measure the ingress-egress network traffic in our load estimation algorithm. Although in our network each router is equipped with only 2 radios, the overall network can utilize more channels with intelligent channel assignment to every link. This is the fundamental reason for non-linear improvement in throughput with respect to the increase in number of radios per node.

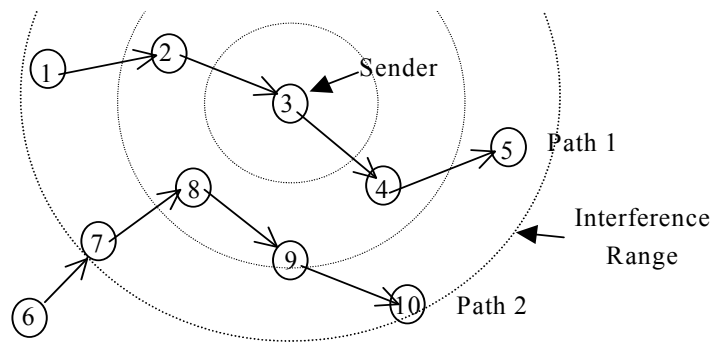

Figure 1. Interference in wireless communication. 


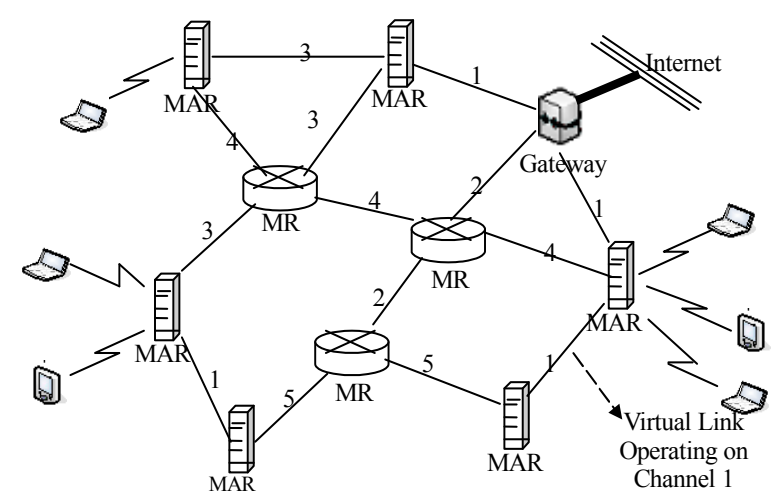

Figure 2. Architecture of multi-radio multi-channel WMN.

\section{Design of Channel Assignment}

The goal of channel assignment in multi-radio WMNs is to bind each radio to a channel in such a way that the available bandwidth on each link is proportional to its expected load. The link here means direct communication on a channel between the routing pair. In this section, we describe the load estimation and channel assignment algorithms.

\subsection{Traffic Measurement}

Optimizations of channel assignment using load estimation require knowledge of network traffic information, so we propose to measure the end-to-end traffics between mesh access routers. The traffic measurement procedure is described as follows:

At first, each mesh access router (including the gateway) measures its ingress-egress flows by probing data periodically (the interval is set to $10 \mathrm{~s}$ in simulations). For convenient expression, we use the access router to indicate both the mesh access router and the gateway in the rest of this paper. Then each access router aggregates its ingress-egress flows and sends the information to the gateway. (The gateway is used as the computation center since it owns the most powerful capacity.)

After receiving the flow information, the gateway calculates the end-to-end traffic value between every pair of access routers by further aggregate the flow information. In this way, the real time value of the end-to-end traffic between each pair of access routers is measured at each echo interval. However, since what the quasi-static algorithm needed is a long-term measured traffic, the gateway performs an exponentially weighted moving average (EWMA) of each end-to-end traffic load to get an approximate long-term traffic. That is:

$$
T(s, d)=\alpha T_{\text {old }}(s, d)+(1-\alpha) T(s, d)
$$

where $T(s, d)$ denotes the end-to-end traffic between access router pair $s$ and $d$. In simulations, the smoothing factor $\alpha=0.7$.

\subsection{Initial Expected Load}

Having the knowledge of the end-to-end traffics, the gateway estimates the expected load on each wireless link and assigns channels to links in order of the expected loads. The gateway is required to perform a new estimation of the expected load either when it receives the traffic information for the first time, or when the difference between the new traffic and the last one is large enough.

To initial the load estimation, we assume that there is a link between each pair of routers in direct communication range, and each end-to-end traffic load is equally divided among all the paths with the least hops between the pair of access routers. (Note that this link won't really exist if there is no common channel assigned to the pair of routers on this link.)

If the number of all shortest paths between node $s$ and $d$ is $P(s, d)$, and in those paths there are $P_{i}(s, d)$ paths passes link $i$, then the initial expected load for link $i$ to carry is calculated as follow:

$$
\varphi(i)=\sum_{s, d} \frac{P_{i}(s, d)}{P(s, d)} T(s, d)
$$

Here we only count the shortest paths because the path with less hops always have much better performance compared with longer paths in multi-hop wireless networks if they all have enough bandwidth.

\subsection{Channel Assignment}

Having knowledge of the expected loads on all network links, we start to assign channels to links as follows:

At first, all links are sorted by their expected loads. Since links expected to carry higher traffic load should be given more bandwidth, the link with the most expected load is prior to other links in choosing channels. Assume every node is equipped with $q$ radios and node $a$ and $b$ is connected by link $i$. There are three conditions for choosing a channel to link $i$ :

1) If both of node $a$ and $b$ have used less than $q$ radios, then choose a channel with the least interference with link $i$. (The chosen channel should also be different from the used channels of $a$ and $b$.) Meanwhile, update the channel lists of node $a$ and $b$.

2) If one of the two nodes (for example $a$ ) has used $q$ channels, then choose a channel from the channel assignment list of node a with the least interference with link $i$. Meanwhile update the channel list of node $b$.

3) When both of node $a$ and $b$ have used $q$ channels: if there exists a common channel between node $a$ and $b$, then assign this channel to link $i$; else, choose one channel from the list of node $a$ and $b$ respectively and merge the two channels to one. Meanwhile update the channel 
lists of all nodes which connect to node $a$ or $b$ using the two channels directly or indirectly.

When link $i$ has been assigned a channel, we remove it from the unassigned link list. As a result, the link with lower expected load now owns the priority to choose channel next. This continues until all network links have been visited, which is denoted as a cycle of channel assignment.

\subsection{End or Feedback}

After a cycle of channel assignment for all links, we need to judge whether current channel assignment satisfies all bandwidth requirements. If so, we terminate the whole procedure and output the channel assignment results, or we feedback the expected link loads under new channel assignment to find a better channel assignment.

It's easy to see that, if the available bandwidth on each link is more than the traffic load it's expected to carry, no congestion will occur. So at first we need to estimate the capacity for each link in the network. However, in wireless networks, channels are shared by all links in the same interference range. So when estimating the usable capacity of a link, we should consider all traffic loads in its interference range. According to the channel assignment rules, the higher load a link is expected to carry, the more bandwidth it should get. On the other side, the higher loads its interfering links are expected to carry, the less bandwidth it could obtain. Thus, the link capacity should be proportional to its traffic load, and be inversely proportional to all other interfering loads. So the capacity for a link $i$ is given by:

$$
C(i)=\frac{B^{*} \varphi(i)}{\sum_{j \in \operatorname{Int}(i)} \varphi(j)}
$$

where B is the channel bandwidth and $\operatorname{Intf}(i)$ stands for the set of links in the interference range of $i$ including itself.

Then the residual capacity of link i can be obtained as below:

$$
R C(i)=C(i)-\varphi(i)
$$

We use the minimal residual capacity of all links on a path as the available bandwidth for this path. Then we consider the bandwidth requirement of an end-to-end traffic is satisfied if there exists a path with its available bandwidth more than the required traffic value.

At the start of the traffic allocation, the initial $R C(i)$ of each link is set to the expected capacity $C(i)$, and the expected load $\phi(i)$ of each link is set to 0 . When a path is chosen to carry an end-to-end traffic, all links on the path reduce their residual capacities and increase their expected loads by the allocated traffic value.
Assume there are totally $\mathrm{N}$ end-to-end traffics with respectively the value of $\mathrm{T}_{\mathrm{n}}(n=1 \ldots, N)$. We choose path $\mathrm{P}$ with the maximal available bandwidth among all the paths with both the least hop and enough available bandwidth for traffic $T_{n}$, which is because the WCETT path metric used in multi-channel routing protocol prefers to choose high throughput paths in multiple channels. When a path is chosen for traffic $T_{n}$, there are two conditions when allocating traffic to this path:

1) If $T_{n} \leq \min _{i \in P}(R C(i))$, we can allocate the whole traffic $T_{n}$ to path $P$. So we decrease the residual capacity and add the expected load of each link i on path $P$ by $T_{n}$. We also decrease the total unallocated traffic by $T_{n}$ for a later comparison of each iteration.

2) If $T_{n}>\min _{i \in P}(R C(i))$, we can only allocate $\min _{i \in P}(R C(i))$ traffic to path P. So we decrease the residual capacity and add the expected load of each link $i$ in path $\mathrm{P}$ by $\min _{i \in P}(R C(i))$. We also decrease the total unallocated traffic value by $\min _{i \in P}(R C(i))$.

When all the $\mathrm{N}$ traffics have been checked, we terminate the whole algorithm if the total unallocated traffic equals to 0 . Or, we compare the total unallocated traffic of this cycle to the last one. If the unallocated traffic of this cycle is no less than the last one, it means no improvement is made and we also terminate the whole process. Otherwise, we feedback the new expected link loads to the channel assignment for a better scheme.

After the whole algorithm ends, the gateway broadcasts the channel assignment results. Then each mesh router adjusts its radios to the assigned channels when they receive the results.

If we combine the traffic measurement, load estimation and channel assignment, the whole algorithm process can be depicted as in Figure 3.

\section{Simulation Analysis}

To evaluate the performance of our channel assignment, we run simulations using ns-2. We use the IEEE 802.11 MAC protocol with RTS/CTS enabled. The AODV protocol is used as the routing protocol for the single-radio IEEE 802.11 network simulations. We modify the AODV protocol using the WCETT metric, a prevailing path metric, as our multi-radio routing protocol. The topology of all networks in the simulations is a 25 -node square grid. To simplify and generalize the simulation, we configure the center node as the gateway and all the other 24 nodes as mesh access routers. The bandwidth of each channel is 2 Mbps. The ratio between interference and communication range is 2 and all nodes in multi-channel networks are equipped with 2 radios. 


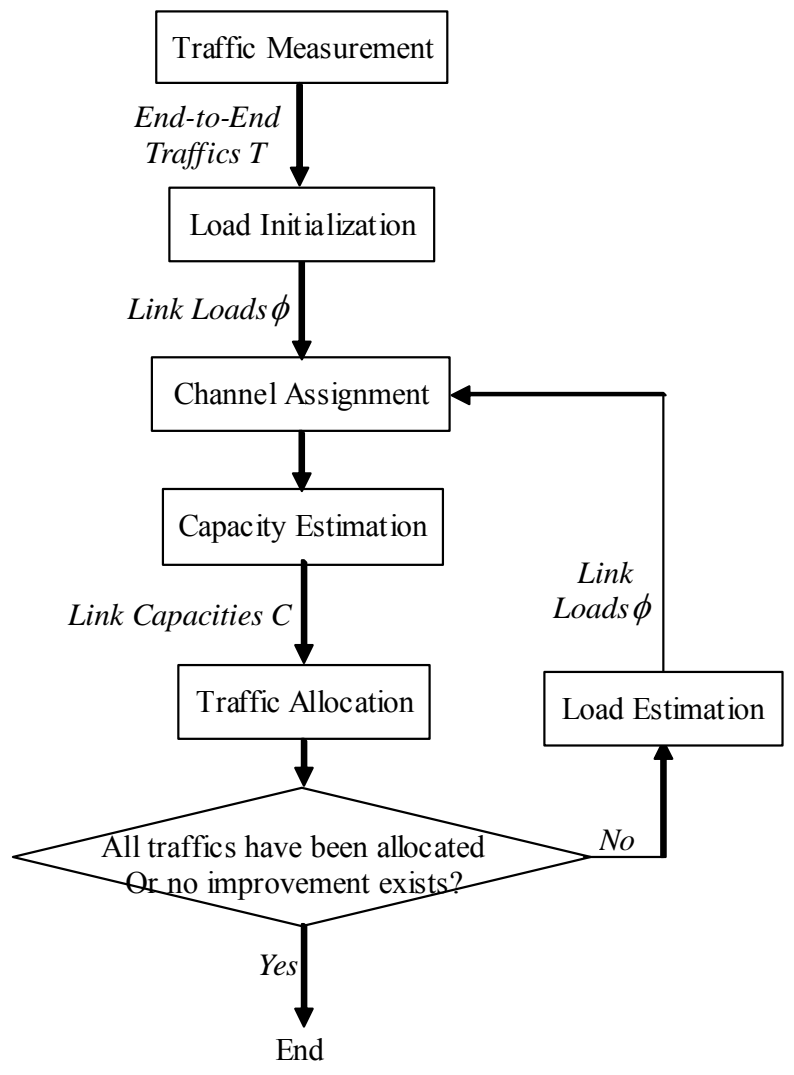

Figure 3. The whole algorithm process.

\subsection{Static Traffic Simulation}

\subsubsection{Flows Simulation}

At first, we randomly choose 10 pair of nodes from the 25 nodes and assign each pair with a different CBR UDP flow. The rate of each flow is chosen randomly between 0 and $0.8 \mathrm{Mbps}$. The packet size is 1000 bytes and flows run for 100 seconds. We test our channel assignment algorithm in a 5-channel network with the 10 flows. To have a good comparison, we also run the same flows in a standard IEEE 802.11 network and a 2-radio 2-channel network. The two networks both needn't any channel assignment and the 2-channel network simulation is just the original WCETT multi-radio routing simulation. We evaluate the improvement of network performance by comparing the aggregate throughputs, the average packet delays and the average packet drop probabilities of the 3 networks.

The aggregate throughputs of the 3 networks are shown in Figure 4. The standard 802.11 network throughput is quite low and the 2-channel network throughput is about twice of the standard 802.11 network. Although the improvement is significant, we can see both the two network throughputs suffer sharp vibration. This is because all network nodes using common channels is quite easy to bring great interference. While in the 5-channel network, we can efficiently limit interference to several small areas using our channel assignment. So the network throughput is quite stable and the value of the throughput also increases a lot.

The average packet delay of each flow is shown in Figure 5. We draw the delays of flows that haven't received any data packet successfully in the whole simulation to the max value of the $y$ axis. We can see the 1-channel network performs badly with 1 flow receiving no packet. Although all flows in the 2-channel network can transport data, the average packet delays for most flows are quite large and exceed $0.5 \mathrm{~s}$. The maximal average packet delay of the 2-channel network is up to $1.83 \mathrm{~s}$. In the 5-channel network, the average packet delays are much smaller. The maximal delay of all flows is $1.15 \mathrm{~s}$ and there are 7 flow delays are below $0.5 \mathrm{~s}$. This further proves that under our channel assignment, the mesh network can efficiently utilize 5 or even more channels with only 2 radios.

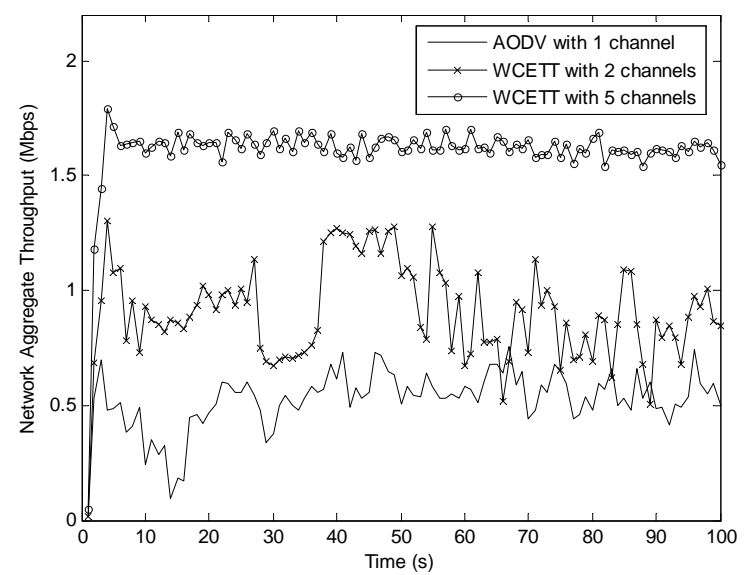

Figure 4. The aggregate network throughput for 10 flows.

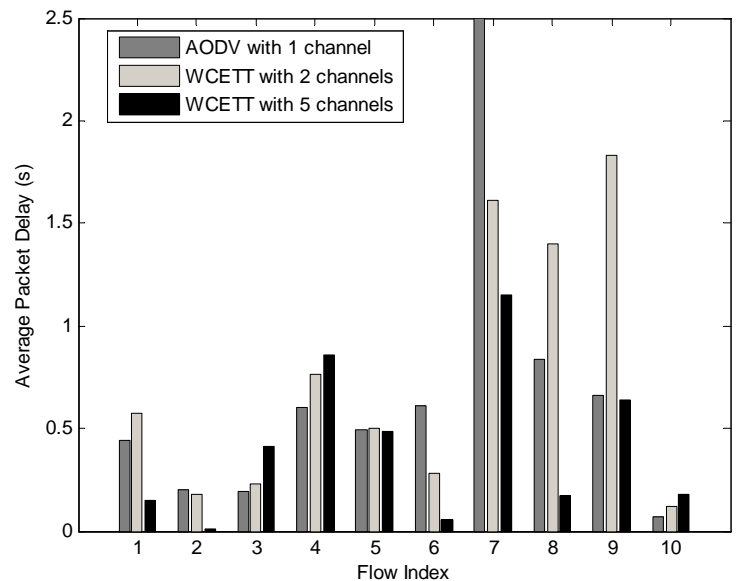

Figure 5. The average packet delay for 10 flows. 
We also compare the average packet drop probability of each flow in Figure 6. The probabilities for the previous two networks are all quite large. In the 5-channel network, the probabilities of 4 flows are quite small with values below 4\%. Although the ones for the other flows are large, but they have decreased a lot compared to the previous two networks.

\subsubsection{Flows Simulation}

To derive the network to saturation, we randomly choose 20 pair of nodes from the 25 nodes and run the same simulations in the above 3 networks. The aggregate throughput of the 3 networks is shown in Figure 7. From Figure 7 we can see the throughput gain for 2-channel network is not significant under the heavy traffic. While both the value and the stability of throughput for 5-channel network get further significant increase, which is due to the sufficient bandwidth brought by efficient utilization of multiple channels.

The average packet delay of each flow is shown in Figure 8 . We can see that in the 1-channel network, 4 flows received no packet in the whole simulation. In the 2-channel network, the average packet delays for most flows are also quite large with the maximal average delay up to $2.32 \mathrm{~s}$ for the 19th flow. While in the 5-channel network, the average packet delays for most flows are below $0.5 \mathrm{~s}$ and the maximal one is only $0.96 \mathrm{~s}$.

We compare the average packet drop probability of each flow in Figure 9. The average packet drop probabilities for all the three networks are all quite large because of the heavy traffic. While compared to the two networks without channel assignment, the average packet drop probability for the 5-channel network is still much smaller and the average drop probability of $9^{\text {th }}$ flow is nearly 0 , which means it has almost transmitted all the data packets successfully.

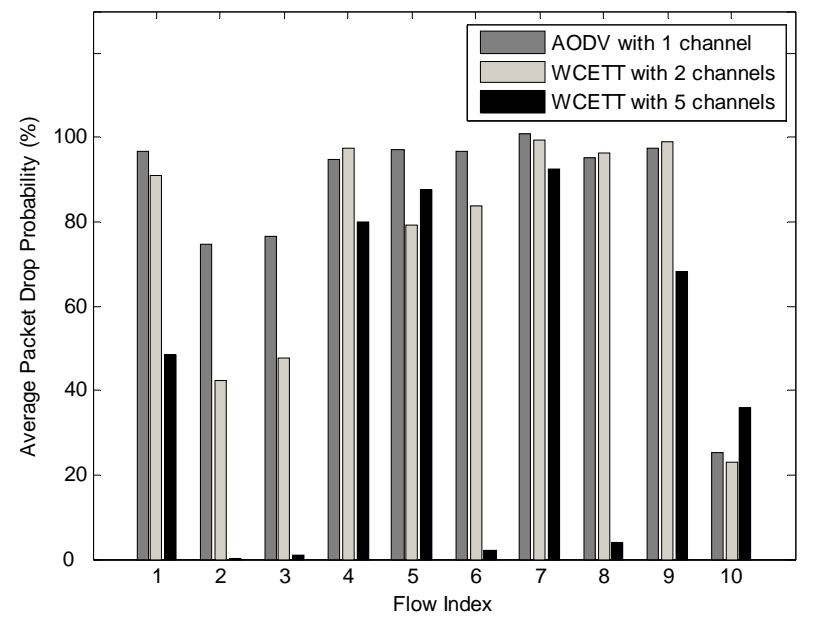

Figure 6. The average packet drop probability for 10 flows.

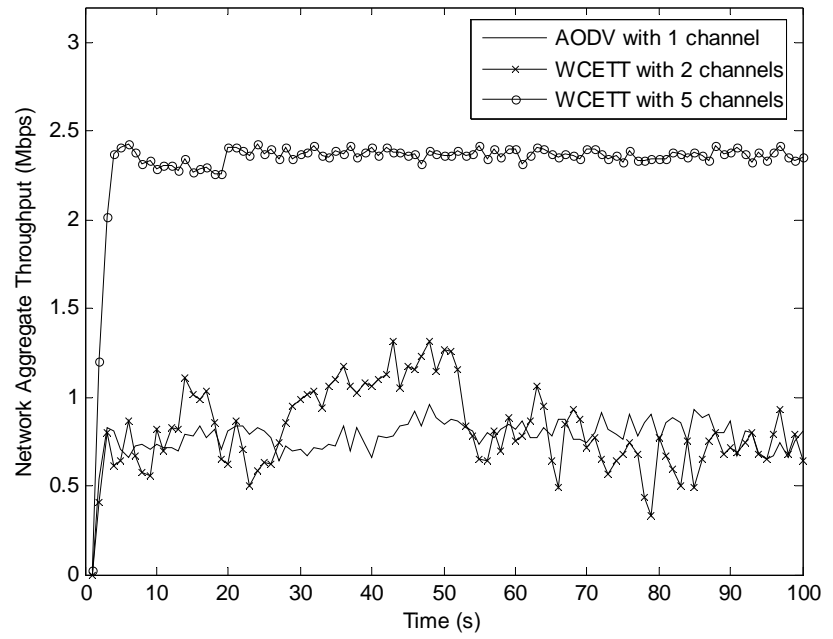

Figure 7. The aggregate network throughput for 20 flows.

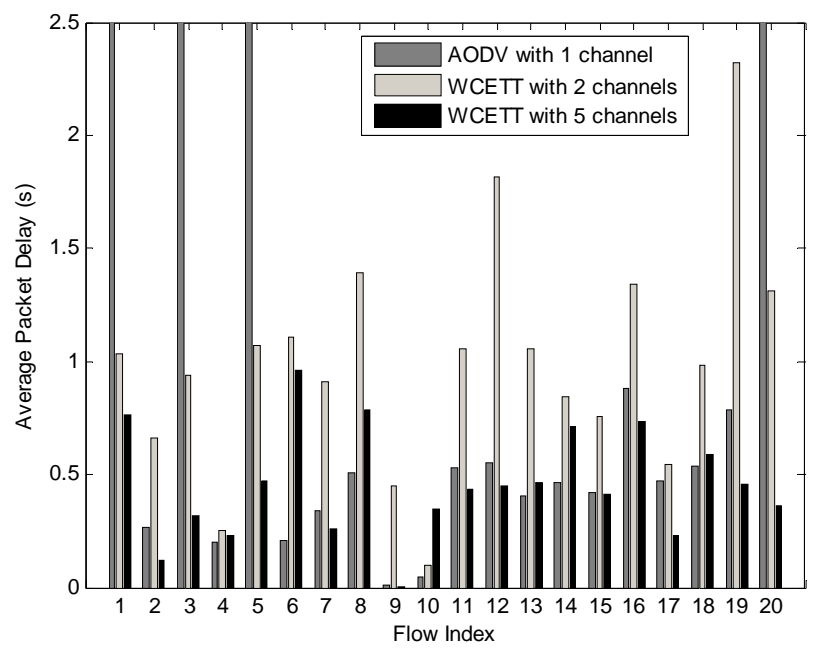

Figure 8. The average packet delay for 20 flows.

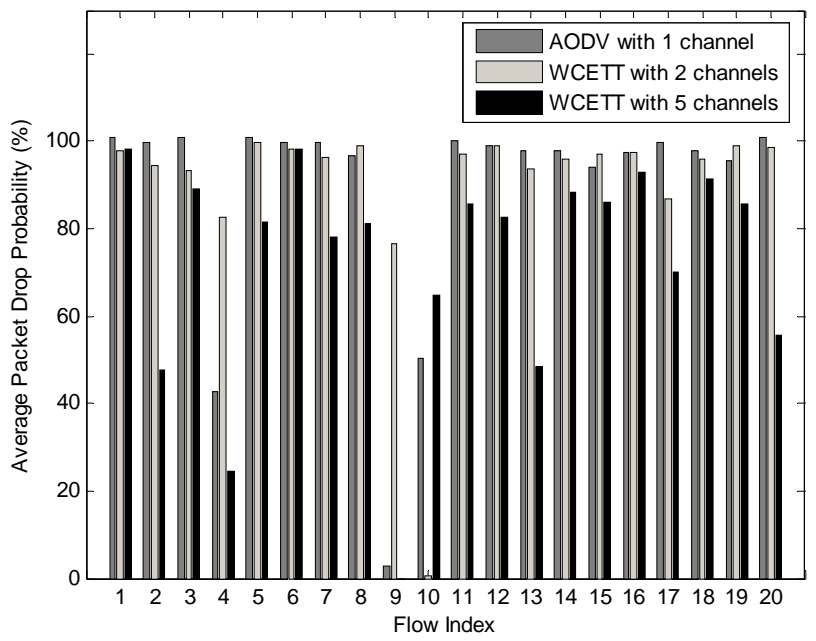

Figure 9. The average packet drop probability for 20 flows. 
Table 1. Comparison of the average aggregate throughput.

\begin{tabular}{ccccccc}
\hline & AODV & WCETT & \multicolumn{3}{c}{$\begin{array}{c}\text { WCETT + Channel } \\
\text { Assignment }\end{array}$} \\
\cline { 3 - 6 } $\begin{array}{c}\text { Avgrage } \\
\text { Througput } \\
\text { (Mbps) }\end{array}$ & 1 channels & 2 channels & $\begin{array}{c}3 \text { chan- } \\
\text { nels }\end{array}$ & $\begin{array}{c}\text { n chan- } \\
\text { nels }\end{array}$ & nels chan- \\
\hline 10 flows & 0.525 & 0.903 & 1.231 & 1.437 & 1.608 \\
20 flows & 0.777 & 0.814 & 1.448 & 2.080 & 2.323 \\
\hline
\end{tabular}

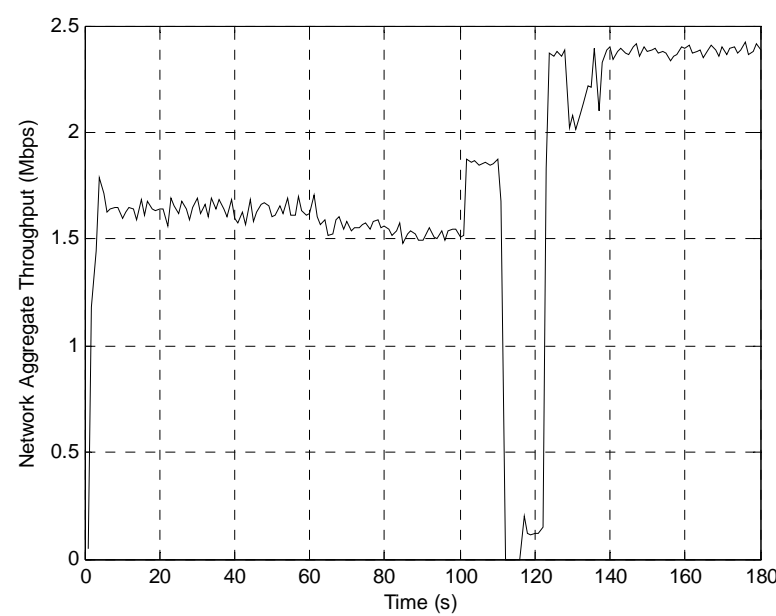

Figure 10. Impact of traffic change on aggregate network throughput.

\subsubsection{The Average Aggregate Throughput Comparison}

Without loss of generality, we also run the 10 and 20 flows respectively in the networks with 3 and 4 channels, using the proposed channel assignment. We list the average aggregate throughputs of the 5 networks with 2 different traffics in Table 1. We see that the network throughput increases with more channels assigned to the network. While the improvement can't be achieved as high times as the number of channels, due to the unneglectable management packets and probing packets for both the WCETT path metric and traffic measurement.

\subsection{Varying Traffic Simulation}

To evaluate the impact of traffic change on network performance, we randomly choose 3 flows in the 10-flow scene and vary their sending rates in the simulation. Flows run for 180 seconds. At the $60^{\text {th }}$ second, we increase the sending rate of a flow by $0.2 \mathrm{Mbps}$. Then at the $80^{\text {th }}$ second, we increase the sending rate of another flow by $0.4 \mathrm{Mbps}$. At the 100 th second, we decrease the sending rate of the third flow by $0.4 \mathrm{Mbps}$. After this, the traffic changes are large enough for the gateway to reassign channels. Because the traffic measurement interval is 10 seconds, the gateway should detect the traffic change and start to reassign channels at the $110^{\text {th }}$ second. Besides, each node need to fresh its routing table since the network channel assignment has changed. So we purge the history information of the WCETT path metric in each node after every new channel assignment.

Figure 10 shows the serial changes of aggregate network throughput by varying the flow sending rates. We can see from the $60^{\text {th }}$ second to the $110^{\text {th }}$ second, the aggregate network throughput changes slightly after each variation of flow sending rate and at last stays around $1.85 \mathrm{Mbps}$. Then at the $110^{\text {th }}$ second, the aggregate throughput suddenly decreases to 0 , which means the network begins to reassign channels. After about 15 seconds vibration, the aggregate throughput comes back to near $2.4 \mathrm{Mbps}$ which means the new channel assignment as well as the WCETT path metric routing have terminated.

Compared to the last $1.85 \mathrm{Mbps}$ throughput before channel reassignment, the network now owns a much better bandwidth assignment. In addition, the channel reassignment and routing together cost only about 15 seconds. So we can say our channel assignment combining with the WCETT path metric routing provides a good choice for the multi-radio WMNs.

\section{Conclusions}

This paper formulated the channel assignment problem in multi-radio multi-channel WMNs. Since the backbone of WMN is an infrastructured network, we assume the mesh routers are stationary and each of them is equipped with 2 radios. Based on network traffic measurement and the load estimation of wireless links, we present a centralized quasi-static channel assignment with the objective of minimizing network interference, which as a result greatly improves network capacity. Extensive simulations show that the proposed scheme is highly responsive to varying traffic conditions, and the network performance under the channel assignment significantly outperforms the single-radio IEEE 802.11 network as well as the 2-radio WMN with static 2 channels.

\section{References}

[1] Mesh Networks Inc. http://www.meshnetworks.com.

[2] I. F. Akyildiz, X. D. Wang, and W. L. Wang, "Wireless mesh networks: A survey," Computer Networks, Vol. 47, No. 4, pp. 445-487, 2005.

[3] Mesh Networking Forum, "Building the business case for implementation of wireless mesh networks," Mesh Networking Forum 2004, San Francisco, CA, October 2004. 
[4] R. Chandra and P. Bahl, "MultiNet: Connecting to multiple IEEE 802.11 networks using a single wireless card," INFOCOM, Vol. 2, pp. 882-893, 2004.

[5] I. Wormsbecker and C. Williamson, "On channel selection strategies for multi-channel MAC protocols in wireless ad hoc networks," IEEE Conference on Wireless and Mobile Computing, Networking and Communications (WiMob’2006), pp. 212-220, 2006.

[6] J. So and N. Vaidya, "Multi-channel MAC for ad hoc networks: Handling multi-channel hidden terminals using a single transceiver," MobiHoc'04, May 24-26, 2004.

[7] A. Raniwala, K. Gopalan, and T. Chiueh, "Centralized channel assignment and routing algorithms for multichannel wireless mesh networks," ACM Mobile Computing and Communications Review, Vol. 8, No. 2, pp. 50-65, 2004.

[8] J. Tang, G. Xue, and W. Zhang, "Interference-aware to- pology control and QoS routing in multi-channel wireless mesh networks," ACM SIGMOBILE, Urbana-Champaign, IL, pp. 68-77, 2005.

[9] A. Subramanian, H. Gupta, and S. R. Das, "Minimum-interference channel assignment in multi-radio wireless mesh networks," Proceedings of 4th Annual IEEE Communications Society Conference on Sensor, Mesh and Ad Hoc Communications and Networks ( SECON'07), pp. 481-490, June 18-21, 2007.

[10] “ns-2 simulator," http://www.isi.edu/nsnam/ns.

[11] R. Draves, J. Padhye, and B. Zill, "Routing in multi-radio, multi-hop wireless mesh networks," in Proceedings of ACM MOBICOM, pp. 114-128, September 2004.

[12] C. Perkins, E. Royer, and S. Das, "Ad hoc on demand distance vector (AODV) routing," IETF Internet Draft, draft-ietf-manet-aodv2-10.txt, January 24, 2002. 